\title{
FRAGILIDADE DE IDOSOS RIBEIRINHOS AMAZÔNICOS: DAS TRAJETÓRIAS METODOLÓGICAS AOS DESAFIOS EM SAÚDE PÚBLICA
}

Rodolfo Gomes Nascimento

Universidade Federal do Pará - UFPA Brasil.

Ronald Oliveira Cardoso

Universidade Federal do Pará - UFPA Brasil.

Denise da Silva Pinto

Universidade Federal do Pará - UFPA Brasil.

Celina Maria Colino Magalhães

Universidade Federal do Pará - UFPA Brasil.
RESUMO: Este estudo tem como objetivo descrever as particularidades teórico-metodológicas e discutir as experiências vivenciadas no processo de pesquisa sobre fragilidade biológica e condições de saúde de idosos ribeirinhos amazônicos. A pesquisa descritiva, de abordagem qualiquantitativa, realizada em 19 ilhas do município de Cametá, Pará, envolveu 108 idosos e permitiu alcançar tanto as proposições diretas sobre os marcadores de fragilidade e de saúde multidimensional, quanto os aspectos subjetivos referentes às particularidades do contexto histórico-cultural. A trajetória percorrida pelos pesquisadores nesse desafio foi marcada, especialmente, por uma arreigada estruturação metodológica, direcionada ao contexto peculiar da pesquisa, desde a inserção ecológica dos pesquisadores, ponto-chave para a concretização do arcabouço metodológico e do período de coleta de dados até a elaboração das propostas norteadoras em saúde pública considerando, sobretudo, as particularidades dos modelos de cuidado vigentes nessas comunidades amazônicas.

PALAVRAS-CHAVE: Métodos; Pesquisa empírica; Idoso fragilizado; Amazônia; Características culturais.

\section{WEAKNESSES OF ELDERLY PEOPLE LIVING NEAR THE AMAZON RIVERS: FROM METHODOLOGICAL TRAJECTORIES TO CHALLENGES IN PUBLIC HEALTH}

\begin{abstract}
Experiences lived during the research on the biological fragility and health conditions of elderly river people on the Amazon are described. Descriptive, qualitative and quantitative research in 19 islands in Cametá, Pará, Brazil, involved 108 elderly people and attained direct propositions on fragility markers, multidimensional health, and the subjective aspects on particulars within the historical and cultural context. Researchers' trajectory was marked by strict methodology, foregrounded on the peculiar context of research ranging from the ecological insertion of the researchers, a key point for the materialization of the methodological design. It also took into account the period of data retrieval until the elaboration of proposals in public health, with special attention to care models in Amazon communities.
\end{abstract}

KEY WORDS: Methods; empirical research; Fragilized elderly people; Amazon; Cultural characteristics. 


\section{INTRODUÇÃO}

Este artigo resultou do processo de construção da tese intitulada "NN1", desenvolvida junto ao Grupo de Estudo "NN2"**, na linha de pesquisa "Processos Evolutivos e Comportamento Humano" do Laboratório de Estudos sobre Desenvolvimento (LED), vinculado ao "NN3"*** A pesquisa de abordagem qualiquantitativa teve como objetivo central investigar as interações entre os indicadores de fragilidade biológica e condições de saúde de idosos em contexto ribeirinho amazônico. Constituiu, portanto, a primeira pesquisa populacional com idosos ribeirinhos amazônicos sobre a temática, incluindo os aspectos biopsicossociais e ecológicos.

Dada a complexidade da questão em foco, compreendeu-se a necessidade de se utilizar um arcabouço metodológico particular para a produção dos dados, que permitisse alcançar os aspectos relacionados à fragilidade, alinhados aos conceitos já bem delineados na literatura nacional ${ }^{2-3}$ e internacional ${ }^{4-6}$, mas que fosse adequado ao contexto de escolha e pudesse permitir maior aprofundamento temático sobre saúde, imbuído de significações, valores e crenças desses idosos.

Desse modo, o longo processo dessa pesquisa transcorreu em fases distintas, como se dá na maioria das pesquisas realizadas em contextos ecológicos diferenciados aos urbanos. As fases prévias foram essencialmente dedicadas ao delineamento dos cuidados conceituais e metodológicos e à inserção ecológica dos pesquisadores nas comunidades ribeirinhas. Em sequência, os pesquisadores dedicaram-se à elaboração do protocolo de pesquisa e coleta de dados. Por último, o desafio envolveu a discussão dos principais resultados alcançados e as proposições em saúde pública.

Em conformidade com o escopo da psicologia do desenvolvimento e considerando as particularidades tradicionais do contexto e do grupo de investigação inseridos na pesquisa, elencou-se como ferramenta teórica o paradigma de desenvolvimento ao longo da vida - Life-S$\operatorname{pan}^{7-8}$, tanto para a fundamentação teórico-metodológica

\footnotetext{
" NN1 Fragilidade e Condições de Saúde de Idosos Ribeirinbos da Amazônia: Indicadores epidemiológicos e aspectos subjetivos

** NN2 Grupo de Estudo pesquisa Envelhecimento na Amazônia (GEEA)

**** NN3 Programa de Pós-graduação em Teoria e Pesquisa do Desenvolvimento
}

quanto para as análises e discussões dos dados evidenciados. A rigor, os objetos de pesquisa foram abordados nessa perspectiva, por a mesma ser contextualista-dialética, e por assumir o desenvolvimento/envelhecimento como um processo complexo, multidimensional e multidirecional que se estende pela vida toda, sendo determinado não só por fatores biológicos, psicológicos e sociais, mas também pelo contexto histórico-cultural ${ }^{7,8}$.

Tendo em conta a importância da temática e a escassez de dados descritivos na literatura, a proposição do presente estudo é descrever as experiências vivenciadas durante o processo de pesquisa de doutorado, enfocando especificamente as particularidades e os desafios dos caminhos metodológicos traçados para a investigação da fragilidade biológica em idosos ribeirinhos amazônicos.

\section{MÉTODO}

Trata-se de um estudo de campo, do tipo descritivo e abordagem qualiquantitativa. Toda a trajetória de pesquisa foi percorrida no período compreendido entre janeiro de 2015 e julho de 2017 e o cenário de estudo compreendeu 19 ilhas fluviais do município de Cametá, Estado do Pará.

Diante desse desafio e tendo em vista as peculiaridades socioculturais do contexto de investigação, as estratégias iniciais de pesquisa foram fundamentadas seguindo um delineamento particular que merece ser descrito. Logo na primeira etapa, percebeu-se a necessidade de um período de inserção ecológica dos pesquisadores na referida região das ilhas para legitimar os conhecimentos que seriam alcançados posteriormente. Procedimentos estes cruciais para o desenvolvimento das etapas seguintes do estudo. Para isso, optou-se por utilizar alguns direcionamentos já postulados sobre esta metodologia em pesquisas desenvolvidas em contextos ribeirinhos ${ }^{9-11}$.

Participaram do estudo 108 idosos ribeirinhos. Para o cálculo do tamanho amostral utilizou-se como base a população de idosos residentes nas áreas rurais do município de Cametá ${ }^{12}$, considerando a prevalência de algum domínio de fragilidade biológica, obtida nos estudos FIBRA-BR ${ }^{3}$, uma rede de pesquisa que se dedicou a desenvolver a primeira investigação de natureza multicêntrica de abrangência nacional sobre a síndrome de fragilidade. 
Considerando as dificuldades logísticas inerentes ao contexto ecológico, cabe destacar que somente foi possível recrutar a amostra de idosos por meio da amostragem não probabilística, por conveniência e de forma consecutiva no período de realização do estudo até completar o número previsto de participantes. Desse modo, realizou-se o recrutamento em função, principalmente, da facilidade de acesso ao domicílio e receptividade/disposição dos mesmos para a coleta dos dados.

Quanto aos critérios utilizados, foram incluídos na pesquisa: idosos a partir de 60 anos de idade; residentes em comunidades ribeirinhas em situação de isolamento territorial; e que aceitaram participar voluntariamente da pesquisa e assinaram o Termo de Consentimento Livre e Esclarecido (TCLE). Na intenção de manter uma uniformização na linha de pesquisas sobre fragilidade, optou-se por adotar os mesmos critérios de exclusão postulados por Fried et al. ${ }^{4}$ e Ferrucci et al. ${ }^{5}$.

Para a coleta de dados foi utilizado um protocolo estruturado pelos pesquisadores com base em instrumentos científicos de pesquisas nacionais e internacionais sobre fragilidade, vulnerabilidade e comunidades ribeirinhas $^{3-4,13}$, que incluía seis instrumentos sobre fragilidade biológica, condições de saúde, condições habitacionais e rotina dos idosos ribeirinhos. Cabe ressaltar que todos os instrumentos, desde o mais geral ao mais específico, foram discutidos e treinados pelos pesquisadores.

Inicialmente, os idosos eram visitados e convidados a participar da pesquisa, recebiam informações sobre a natureza e objetivos do estudo e, após aceitarem participar da pesquisa formalmente pela assinatura ou coleta das impressões digitais no TCLE, eram submetidos ao checklist dos critérios pré-estabelecidos para inclusão/ exclusão. A exemplo de outros estudos já realizados ${ }^{3-4}$, após essa etapa realizou-se a avaliação do status cognitivo mediante o Mini Exame do Estado Mental (MEEM), na intenção de garantir a confiabilidade dos dados de autorrelato (Figura 1).

Os idosos que pontuaram abaixo da nota de corte para seu nível de escolaridade foram avaliados de acordo com os dados demográficos e socioeconômicos; indicadores de fragilidade; medidas antropométricas e pressão arterial, e condições habitacionais, correspondentes ao primeiro bloco de coleta de dados. Já os idosos que pon- tuaram acima da nota de corte, realizaram as mesmas avaliações e outras relativas aos dados de autorrelato sobre o grau de satisfação habitacional e indicadores da condição de saúde, correspondentes ao segundo bloco da coleta de dados.

No delineamento da operacionalização do fenótipo de fragilidade biológica dos idosos ribeirinhos utilizamos o modelo postulado por Fried et al. ${ }^{4}$ modificado, composto por cinco critérios (a) autorrelato de perda de peso não intencional no último ano, igual ou superior a $4,5 \mathrm{~kg}$ ou a $5 \%$ do peso corporal; (b) fadiga indicada pelas respostas sempre ou quase sempre a dois itens da Center for Epidemiological Study - Depression (CES-D); (c) baixa força de preensão manual ( Figura 1), avaliada através do dinamômetro do tipo JAMAR, modelo NC $701 / 42$, correspondente aos valores localizados entre os $20 \%$ menores da distribuição das médias das três medidas realizadas, ajustadas por sexo e IMC, adotando-se os pontos de corte propostos por Neri ${ }^{14}$; (d) baixa velocidade da marcha (Figura 1), quando a média de três medidas consecutivas do tempo para percorrer $4,6 \mathrm{~m}$ no plano e em passo usual é superior ao percentil 80 da amostra, médias essas ajustadas por sexo e altura, adotando-se os pontos de corte propostos por Neri ${ }^{14 ;} \mathrm{e}$ (e) baixo nível de atividade física, pontuaram para este critério aqueles cuja classificação no "Questionário de nível de atividade física para idosos CuritibAtiva adaptado"15 estava compatível com o grupo inativo ou pouco ativo.

De acordo com essa proposta de fragilidade, os idosos foram classificados como frágeis, se três, quatro ou cinco itens estivessem presentes; pré-frágeis, se um ou dois itens estivessem presentes; ou não frágeis, se nenhum critério estivesse presente ${ }^{4}$.

Ao final das sessões de coleta, cada idoso recebia aconselhamentos em saúde envolvendo orientações de promoção de saúde e prevenção de agravos na velhice, contemplando especialmente o que foi observado em relação aos principais comprometimentos e necessidades em saúde. Para isso foi utilizado um folheto como tecnologia educativa, elaborado de forma ilustrada para melhor entendimento, considerando a baixa escolaridade da maioria dos idosos (Figura 1).

Este estudo foi aprovado pelo Comitê de Ética em Pesquisa com seres humanos do "NN", segundo o 
parecer de $\mathbf{n}^{\circ}$ "NN", obedecendo às diretrizes e normas regulamentadoras de pesquisa em seres humanos, conforme a resolução no 466/2013 CNS.

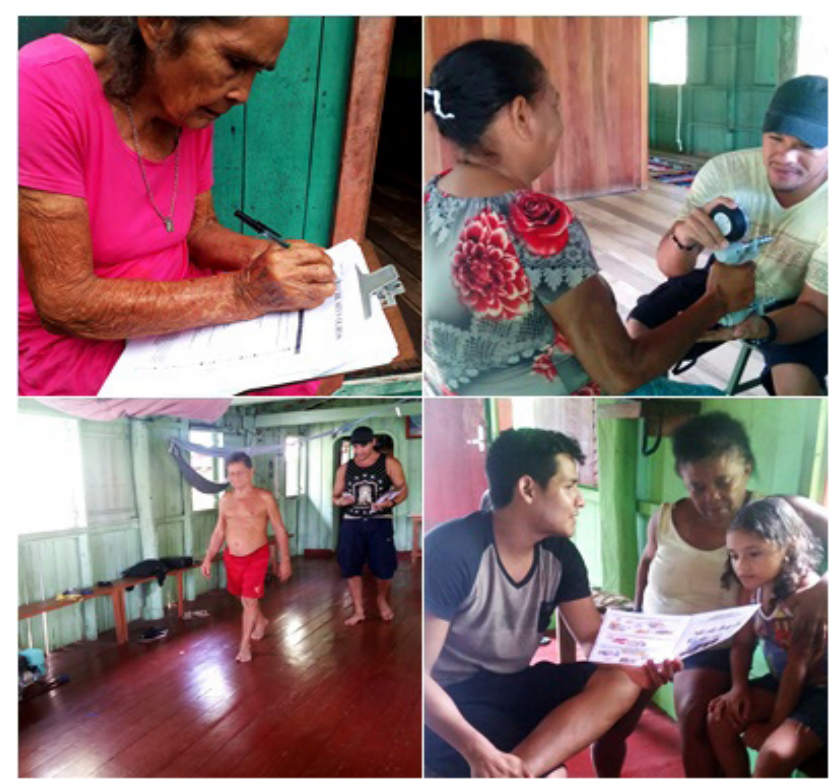

Figura 1. Aplicação do miniexame do estado mental, teste de preensão palmar, teste de velocidade da marcha e aconselhamentos em saúde

Fonte: Do autor (2017)

\section{RESULTADOS E DISCUSSÕES}

Nas últimas décadas o estudo da fragilidade tem despertado crescente interesse na comunidade científica nacional e internacional ${ }^{2,3}$. Em suma, o cenário científico tem postulado dados importantes sobre fragilidade de idosos brasileiros, apontando importantes relações entre pré-fragilidade, fragilidade e variáveis sociodemográficas, o que reforça a necessidade de um direcionamento de pesquisa em cenários onde indivíduos envelhecem em maiores condições de vulnerabilidade ${ }^{3}$. No entanto, estes são predominantemente produzidos em cenários urbanos. Nota-se, portanto, que o caminho do conhecimento sobre essa temática em contextos rurais ainda é pouco percorrido e, especialmente, naqueles tipicamente amazônicos, ainda hoje representam uma lacuna na literatura científica.

Sobre a escolha do contexto ecológico onde foram desenvolvidos os estudos, o município de Cametá, localizado no nordeste Paraense, está distante em linha reta a $156 \mathrm{~km}$ da capital do Estado, Belém ${ }^{12} \mathrm{e}$ concentra a maior parte da sua população nas zonas rurais, abrangendo em grande parte a "região das ilhas". Estas abarcam as áreas de várzea, ou seja, as porções de terras alagadas temporariamente sob a influência direta das águas do rio Tocantins e correspondem a aproximadamente 100 ilhas fluviais potencialmente habitáveis ${ }^{16}$. Tais características o caracterizam como um município amazônico tipicamente ribeirinho e foram decisivas para a escolha desse cenário para a pesquisa, uma vez que haveria grande possibilidade de alcançar o número amostral previsto para os estudos e pelo entendimento de que, no local, existiam idosos essencialmente ribeirinhos com costumes, tradições e crenças culturalmente amazônicas que influenciavam diretamente no cuidado à saúde.

As questões metodológicas iniciais na condução da pesquisa envolveram alguns importantes desafios conceituais, tanto em relação ao constructo de fragilidade, que pudessem abarcar a identificação da higidez física desses idosos ribeirinhos, quanto em relação ao termo "ribeirinho", dado que existem particularidades importantes no cenário amazônico as quais poderiam enviesar os dados coletados.

Após travadas muitas discussões, decidiu-se por utilizar o modelo fenotípico proposto por Fried et al. ${ }^{4} \mathrm{pa}$ ra a definição de fragilidade, ponderando especialmente o fato que este se ancora predominantemente ao caráter físico dessa síndrome. Em adendo, considerando ainda que a maioria das pesquisas sobre a temática tem ampliado as discussões sustentando o caráter multidimensional ${ }^{17}$, o que também está alinhado ao que pressupõe a perspectiva contextualista-dialética, decidiu-se por articular o rastreio físico ao estudo de componentes sociais, clínicos, funcionais, psicológicos, cognitivos, ecológicos, dentre outros.

O reconhecimento da escassez de trabalhos na área que envolvam idosos ribeirinhos com características peculiares amazônicas motivou o estabelecimento de outros cuidados metodológicos iniciais que merecem ser destacados, especialmente para que futuras produções possam estabelecer os mesmos cuidados, evitando confusões teórico-conceituais.

$\mathrm{O}$ primeiro diz respeito à definição do termo "ribeirinho" enquanto norteador da amostra alvo da pesquisa. Partindo de uma definição literal, "ribeirinho" designa indivíduos que andam ou vivem às margens de rios ou ribeiras. No entanto, considerando que os indiví- 
duos a serem investigados deveriam ser diferenciados de qualquer habitante que também mora nas orlas de rios que atravessam grandes centros urbanos dos municípios amazônicos como Manaus, Porto Velho e Belém, foi necessário adotar a definição de Cohen-Carneiro et al. ${ }^{18}$, a qual se refere como "ribeirinho" aquele indivíduo que vive e subsiste fundamentalmente do rio à margem do qual habita, tendo com este uma relação de dependência nas suas necessidades básicas de alimentação, transporte, trabalho e subsistência.

O segundo cuidado e não menos importante, esteve alinhado ao estabelecimento do critério de isolamento territorial das comunidades. Nesta pesquisa, permitiu-se que somente os idosos que possuíam o modo de vida rural-ribeirinho fossem investigados, uma vez que na região amazônica há comunidades próximas de centros urbanos cujo seus habitantes estabelecem maior parte dos seus modos de vida nos espaços urbanos. Portanto, ficou estabelecido que seriam visitadas somente as localidades que tinham pouca proximidade com as sedes urbanas dos distritos do município e com acesso somente por via hidroviária, através de embarcações motorizadas como as denominadas "rabetas" e "rabudos", meio de transporte tradicional da região.

Diferentemente das inúmeras pesquisas nacionais e internacionais sobre fragilidade de idosos em contextos rurais e em regiões em situação de vulnerabilidade socias ${ }^{19-21}$, na presente pesquisa houve a necessidade de se realizar a inserção ecológica dos pesquisadores no contexto de estudo, outra importante questão metodológica envolvida previamente à coleta de dados. Articularam-se inicialmente contatos com nativos das regiões das ilhas do município de Cametá que resultaram em várias viagens ao longo do primeiro semestre de 2015. Nessa oportunidade, os pesquisadores visitaram comunidades ribeirinhas localizadas em sete ilhas para apropriação de informações de viabilidade como, por exemplo, a possibilidade de se estabelecer as bases para a hospedagem durante a coleta dos dados da pesquisa.

Além disso, outras importantes estratégias de operacionalização foram possíveis, dentre elas: o estabelecimento dos processos de aproximação do contexto, a qual permitiu ampliação do olhar em torno da realidade nesse contexto, apropriando-se das experiências dos des- locamentos nos rios, das ambientações nas estadias, das expressões linguísticas típicas da região e dos costumes tradicionais dos idosos nesse contexto; e a construção de vínculos com a população ribeirinha em geral e com os idosos, efetivada por meio de várias visitas domiciliares intermediadas pelos informantes nativos das localidades, os quais tinham maior proximidade com os moradores.

Vale ressaltar que durante o período de inserção ecológica foi aplicado um inventário de rotinas, desenvolvido com base no trabalho de Silva et al..$^{13}$ e adaptado para esse estudo tomando como referência aspectos ligados à pessoa idosa e ao seu modo de vida ribeirinho; coletado material audiogravado, bem como registros fotográficos. Além disso, utilizamos a técnica observação-participante, contemplada por meio da utilização do diário de campo, seguindo os norteamentos para sua utilização em pesquisas em contextos ribeirinhos ${ }^{10}$.

Por meio desta abordagem metodológica, no transcorrer desta etapa foi possível registrar, sobretudo, informações no que diz respeito ao processo de saúde-doença dos idosos e do modo de vida neste contexto ecológico, na intenção de garantir uma visão ampliada da temática, possibilitando maior familiaridade com o problema do estudo. Assim, pôde-se aprofundar a compreensão dos aspectos centrados na realidade dessas comunidades ribeirinhas e efetivar as adaptações de alguns instrumentos.

De fato, cabe a ressalva que essa estratégia foi extremamente útil, pois que os pesquisadores mergulhassem no universo amazônico vivenciado pelos idosos, proporcionando um envolvimento mútuo e valoroso para a concretização das etapas seguintes de investigação. Registra-se, portanto, que tal procedimento merece reprodutibilidade por quem se propõe pesquisar em contextos diferenciados, principalmente aqueles que apresentam dificuldades operacionais face o distanciamento geográfico.

Sobre o período de coleta dos dados propriamente dito, levando em consideração o distanciamento das comunidades ribeirinhas da capital Belém, a inexistência de um transporte regular para o deslocamento da equipe até o local e o custo envolvido tanto para o deslocamento quanto para a manutenção da equipe nas comunidades, a opção metodológica mais adequada foi viabilizar esta 
etapa por meio de coletas periódicas intensivas.

Todos os procedimentos referentes a essa etapa foram realizados no próprio domicílio dos idosos, em sessão única. Portanto, vale registrar que esse processo foi árduo, contudo, logo de início, pudemos constatar uma característica bem marcante destas comunidades: sua hospitalidade e disponibilidade para contribuir com todos do grupo de pesquisa. Em cada palafita visitada, os ribeirinhos externavam seu contentamento e sua satisfação por reconhecer o interesse dos pesquisadores em compreender suas mazelas da atenção à saúde. Neste ponto, cabe destacar o papel dos informantes nativos, essenciais para intermediar essas aproximações.

Com relação ao protocolo para a coleta de dados da pesquisa, alguns instrumentos foram compilados em versão original, outros foram ajustados na intenção de adequar aos objetivos propostos, com base nos registros preliminares produzidos na inserção ecológica dos pesquisadores. Neste sentido, as adequações levaram em consideração, especialmente a linguagem mais apropriada a ser utilizada junto aos participantes, a logística do contexto investigado, entre outros aspectos.

Sobre o MEEM, tanto a heterogeneidade sociocultural amazônica, quanto algumas recomendações publicadas sobre a importância de se adaptar/adequar o MEEM a populações idosas brasileiras de baixa escolaridade e inseridas em contextos culturais diferenciados ao urbano $^{22-23}$, foram determinantes para a tomada de algumas decisões necessárias.

Considerando o fato de que alguns termos utilizados no MEEM original não seriam bem compreendidos pelos idosos por conta do baixo acesso à educação formal e, também, por não fazerem parte do repertório linguístico típico dessas regiões, foi necessária a adequação do instrumento às especificidades linguísticas e culturais ribeirinhas preservando as intenções da sua versão original. Além disso, considerando os posicionamentos críticos de Macuco et al. ${ }^{24}$, optou-se por utilizar os pontos de corte mais baixos, recomendados por Bertolucci et al..$^{25}$, a exemplo do estudo de Silva ${ }^{25}$ realizado no Estado do Amazonas.

Uma das maiores dificuldades enfrentadas na avaliação da fragilidade dos idosos ribeirinhos foi com relação à avaliação do nível de atividade física destes in- divíduos. Dadas as particularidades do contexto ecológico e sociocultural dessas comunidades, a maioria das atividades desenvolvidas cotidianamente por esses idosos não corresponde àquelas desempenhadas por idosos urbanos ou de zonas rurais de terra firme. Os instrumentos utilizados para este fim em pesquisas já realizadas com idosos rurais da Colômbia ${ }^{17}$, da Coréia ${ }^{19}$ ou com idosos brasileiros em contexto de pobreza ${ }^{20-21}$, ou com idosos amazônicos ${ }^{26}$, os que talvez mais se aproximassem da população-alvo da pesquisa, não são consensuais. Desse modo, os pesquisadores utilizaram outros instrumentos que não o preconizado pela proposta de Fried et $\mathrm{al}^{4}$ para contemplar as particularidades da população estudada.

$O$ instrumento utilizado nesta pesquisa foi validado por Rauchbachet al. ${ }^{15}$. Optou-se pela utilização desse questionário por considerá-lo mais próximo da realidade dos idosos brasileiros e pela facilidade de aplicação. Cabe ressaltar que, pelas particularidades do contexto ribeirinho, adaptações no conteúdo fizeram-se necessárias para apreciar as prováveis atividades desempenhadas pelos idosos nos ambientes domiciliares e sociais ribeirinhos como, por exemplo, capinar, tirar o mato, subir e descer as escadarias, pescar, remar, tomar banho no rio etc. Além disso, todas as pontuações das atividades incluídas foram devidamente ajustadas segundo a recomendação do "Compendium of Physical Activities Tracking Gui$d e^{" 27}$. Por fim, todos esses cuidados metodológicos foram de grande valia, uma vez que com a interpretação dos resultados obtidos com o instrumento pode-se atribuir adequadamente o real nível de atividade física desses idosos.

Lançando o olhar para o eixo central dos estudos, referente à identificação da fragilidade biológica, dos 108 idosos ribeirinhos idosos apenas 9,3\% de idosos frágeis, $38,9 \%$ de idosos pré-frágeis e $51,9 \%$ de idosos não frágeis. A proporção de idosos robustos, que não pontuaram em nenhum critério para a fragilidade, foi o que mais chamou atenção nos resultados evidenciados. Ao passo disso, e considerando todo o conjunto de dados alcançados, confiou-se a proposição de que pelo prisma de análise da perspectiva Life-span ${ }^{7,8}$, apesar da notória vulnerabilidade social dessa população, a ação conjunta entre as dimensões biológicas e sócio-históricas influenciam-se reciprocamente de tal maneira a conferir do ponto de vista físico/ biológico baixas condições de fragilização. 
Procurando estabelecer pontes entre as proposições da pesquisa e os desafios em saúde pública, também foi objetivo do trabalho analisar o comportamento dos marcadores das condições de saúde. Para se alcançar o referido objetivo, a estratégia metodológica utilizada foi o estudo das associações estatísticas pela análise bivariada entre a variável de desfecho e as variáreis independentes. Enfim, foi visto que a fragilidade dos idosos ribeirinhos estava associada às seguintes variáveis: idade mais avançada, circunferência de cintura, razão cintura-quadril, MEEM, Escala de Depressão Geriátrica, uso de medicamentos, polifarmácia, polipatologia, dentre outras ${ }^{1}$.Vislumbram-se, aqui, como pesquisadores a importância dessa etapa da pesquisa que foi exitosa e essencial no cumprimento do retorno social à comunidade pesquisada.

Assim como também foi percebido nas pesquisas de Neri et al. ${ }^{3}$, Reis Junior et al. ${ }^{20}$, Zazzetta et al. ${ }^{21}$ e Sil$\mathrm{va}^{26}$, tanto o contingente de idosos em condição de pré-fragilidade quanto os marcadores associados à síndrome, serviram de alerta para a necessidade de investimentos em saúde pública. No caso da presente pesquisa, tais investimentos devem ser sensíveis às problemáticas gerontológicas próprias do contexto ribeirinho, diferentemente daquelas propostas para idosos inseridos em contextos urbanos, o que os tornam mais desafiadores.

Como última etapa do trabalho, foi elaborado um parecer técnico e encaminhado à secretaria de saúde do município locus do estudo, destacando, sobretudo, o conjunto de medidas sugeridas no corpo da tese com vistas ao cumprimento dos direitos à saúde integral, à equidade e à universalização da assistência, princípios fundamentais do Sistema Único de Saúde.

Destacam-se dentre as propostas, a ampliação da área de cobertura da Estratégia Saúde da Família (ESF) na região das ilhas, que possam consolidar e fortalecer a rede de atenção primária, através de capacitações dos profissionais de saúde, inclusive os que são ribeirinhos e que trabalham como Agentes Comunitários de Saúde, para que possam reordenar suas ações e práticas terapêuticas institucionais, articulando-as com o modelo de suporte terapêutico tradicional de cuidado vigente nesses contextos ecológicos, uma vez que o "benzimento", o "curandeirismo" e a prática de "puxação" geralmente constituem as primeiras opções como forma de enfrentamento aos problemas de saúde nessas comunidades e devem ser consideradas e culturalmente respeitadas.

Dentre as alternativas possíveis, destacou-se a Educação Popular em Saúde (EPS); e por último, porém não menos importante, a efetivação da gestão da fragilidade por meio de modelos assistenciais do idoso ribeirinho na atenção primária efetuada por instrumentos que permitem a identificação e o monitoramento da população de maior risco de fragilização, apontando resultados e classificações imediatas sem a necessidade de equipamentos específicos para isso, como o Índice de Vulnerabilidade Clínico-Funcional-20 (IVCF-20) ${ }^{28}$ e o Vulnerable Elders Survey (VES-13) ${ }^{29}$.

\section{CONSIDERAÇÕES FINAIS}

O processo de construção de pesquisa nos contextos ribeirinhos da região amazônica, cujo distanciamento geográfico dos centros acadêmicos é permeado por importantes desafios, merecem enfrentamentos e notoriedade científica no sentido de garantir reprodutibilidades e aprimoramentos futuros. Desse modo, neste artigo, procuramos descrever os percursos adotados, desde as etapas de idealização temática, passando pela concretização dos resultados até os procedimentos finais de garantia do retorno social no campo da saúde pública, destacando especialmente as riquezas e particularidades dos métodos utilizados e as maiores dificuldades do percurso.

Desenvolver pesquisa em contextos diferenciados ao urbano é sem dúvida desafiador. Durante todo o período de imersão muitos empecilhos atrelados ao critério de isolamento territorial das comunidades tiveram que ser superados, desde a intermediação de contatos para se alcançar as ilhas; o estabelecimento das bases para a hospedagem dos pesquisadores em moradias de nativos da região; a distância entre as moradias por conta da ocupação esparsa nas ilhas; a identificação das residências que tinham pessoas idosas para compor a amostra; a intermediação para que o acesso às moradias fosse permitido; a conciliação de horários disponíveis para a participação na pesquisa por parte dos idosos, dentre outros.

Frente aos resultados alcançados, cabe assinalar algumas questões metodológicas chaves para o sucesso 
desta pesquisa. Por certo, conhecer previamente as regiões e estabelecer vínculos com a comunidade por intermédio de nativos foi assertivo. Além disso, considerando a complexidade da proposta, a utilização de instrumentos adaptados à realidade do contexto ecológico e histórico-cultural de pesquisa foi essencial para a compreensão do objeto de estudo - fragilidade biológica.

Podemos concluir, portanto, que a análise e a discussão dos dados apresentados nos permitiram confirmar as percepções inicialmente empíricas sobre 0 constructo de fragilidade biológica dos idosos que vivem às margens dos rios da Amazônia. Destacamos que tais evidências somente foram alcançadas a partir de um arcabouço metodológico extenso e integrado, o qual abrangeu a investigação das interações entre pessoa-contexto ancoradas aos aspectos histórico-sócioculturais. Sendo assim, apresentou-se como um caminho metodológico possível e válido aos que procuram compreender fragilidade e saúde de pessoas idosas ribeirinhas, sem abrir mão das suas particularidades.

Evidentemente, esta condição relativa ao ótimo desempenho físico não necessariamente está associada a uma representação positiva de saúde, já que frente às especificidades territoriais e culturais existem importantes vulnerabilidades em saúde que se relacionam aos indicadores de saúde e bem-estar na velhice.

\section{AGRADECIMENTOS}

Os autores agradecem ao Programa de Apoio à Pós-graduação da Coordenação de Aperfeiçoamento de Pessoal de Nível Superior (PROAP/CAPES), pelo apoio ao desenvolvimento desta pesquisa.

\section{REFERÊNCIAS}

1. Nascimento RG, Magalhães, CMC. Fragilidade e Condições de Saúde de Idosos Ribeirinhos da Amazônia: Indicadores epidemiológicos e aspectos subjetivos. Belém. Tese [Doutorado em Teoria e Pesquisa do Comportamento] - Universidade Federal do Pará; 2017.

2. Mello AC, Engstrom EM, Alves LC. Fatores sociodemográficos e de saúde associados à fragilidade em idosos: uma revisão sistemática de literatura. Cadernos de Pública. 2014; 30(6): 1-25.
3. Neri AL et al. Metodologia e perfil sociodemográfico, cognitivo e de fragilidade de idosos comunitários de sete cidades brasileiras: Estudo FIBRA. Cadernos de SaúdePública. 2013; 29(4): 778-792.

4. Fried LP et al. Frailty in older adults: evidence for a phenotype. The Journals of Gerontology Series A: Biological Sciences and Medical Sciences. 2001; 56(3): M146-M157.

5. Ferrucci L et al. Designing randomized, controlled trials aimed at preventing or delaying functional decline and disability in frail, older persons: a consensus report. Journal of the American Geriatrics Society. 2004; 52(4): 625-634.

6. Fried LP et al. Untangling the concepts of disability, frailty, and comorbidity: implications for improved targeting and care. The Journals of Gerontology Series A: Biological Sciences and Medical Sciences. 2004; 59(3): M255-M263.

7. Baltes PB. On the incomplete architecture of human ontogeny: Selection, optimization, and compensation as foundation of developmental theory. American psychologist. 1997; 52(4): 366.

8. Baltes PB. Theoretical propositions of life-span developmental psychology: On the dynamics between growth and decline. Developmentalpsychology. 1987; 23(5): 611.

9. Mendes LSA et al. Inserção ecológica no contexto de uma comunidade ribeirinha amazônica. InteramericanJournalofPsychology . 2008; 42(1):1-10.

10. Afonso T et al. O uso do diário de campo na inserção ecológica em uma família de uma comunidade ribeirinha amazônica. Psicologia \& Sociedade. 2015; 27(1): 131-141.

11. Silva SSC et al. Avaliação de Famílias Ribeirinhas: Uma Proposta Adaptada ao Contexto. Gerais: Revista Interinstitucional de Psicologia, 2011; 4(2): 253-263.

12. IBGE. Instituto Brasileiro de Geografia e Estatística. (2010). Censo Demográfico de 2010.

13. Silva SSC et al. Rotinas familiares de ribeirinhos amazônicos: uma possibilidade de investigação. Psic.: Teor. e Pesq. 2010; 26(2): 341-350.

14. Neri AL. Fragilidade e qualidade de vida na velhice. $1^{\mathrm{a}}$ ed. Campinas: Editora Alínea; 2013. 
15. Rauchbach R, Wendling NMS. Evolução da construção de um instrumento de avaliação do nível de atividade física para idosos curitibativa. FIEP Bulletin online. 2009; 79: 543-47.

16. Araújo MPD. A organização do trabalho pedagógico em escolas multisseriadas no Município de Cametá [Dissertação]. Belém (PA): Programa de Pós-Graduação em Educação da Universidade Federal do Pará; 2012.

17. Curcio CL, Henao GM, Gomez F. Frailtyamong rural elderlyadults. BMC geriatrics, 2014; 14(1): 1-9

18. Cohen-Carneiro et al. Oferta e utilização de serviços de saúde bucal no Amazonas, Brasil: estudo de caso em população ribeirinha do Município de Coari. Cadernos de Saúde Pública. 2009; 25(8):1827-1838.

19. Jung, HW et al. Prevalence of frailty and aging-related health conditions in older Koreans in rural communities: a cross-sectional analysis of the aging study of Pyeongchang rural area. Journal of Korean medical Science, 2016; 31(3): 345-352.

20. Reis Junior WM et al. Pré-fragilidade e fragilidade de idosos residentes em município com baixo Índice de Desenvolvimento Humano. Revista Latino-Americana de Enfermagem, 2014, 22(4): 654-661.

21. Zazzetta MS et al. Identifying frailty levels and associated factors in a population living in the context of poverty and social vulnerability. Journal of frailty and aging, 2017, 6(1): 29-32.

22. Brito-Marques PRD, Cabral-Filho JE. Influence of age and scholing on the performance in a modified Mini-Mental State Examination version: a study in Brazil Northeast. Arquivos de Neuro-psiquiatria. 2005; 63(3A): 583-587.

23. Brito-Marques PRD, Cabral-Filho JE. The role of education in mini-mental state examination: a study in Northeast Brazil. Arquivos de Neuro-psiquiatria. 2004; 62(2A): 206-211.

24. Macuco CM et al. Cognição e fragilidade. In: Neri AL, Fragilidade e qualidade de vida na velhice. Campinas, SP: Editora Alínea; 2013. p. 85-98.

25. Bertolucci PH et al. O mini-exame do estado mental em uma população geral: impacto da escolaridade. Arquivos de Neuro-psiquiatria. 1994; 25(1):1-7.

26. Silva KRD. Prevalência de Síndrome da fragilidade em idosos da área urbana do município de Coari-Amazonas: um estudo de base populacional. [Dissertação]. Manaus (AM): Mestrado em saúde, sociedade e endemias na Amazônia da Universidade Federal do Amazonas; 2016.

27. Ainsworth BE et al. Compendium of physical activities: an update of activity codes and MET intensities. Medicine and science in sports and exercise, 2000; 32(9): S498-S504.

28. Moraes END et al. Clinical-Functional Vulnerability Index-20 (IVCF-20): rapid recognition of frail older adults. Revista de saúde pública, 2016, 50(81): 1-10.

29. Maia FOM et al. Adaptação transcultural do VulnerableElders Survey-13 (VES-13): contribuindo para a identificação de idosos vulneráveis. Revista da Escola de Enfermagem da USP, 2012, 46: 116-122.

Recebido em: 27/12/2018 Aceito em: 28/05/2019 